\title{
Nasal Congestion, CTCAE
}

National Cancer Institute

\section{Source}

National Cancer Institute. Nasal Congestion, CT CAE. NCI Thesaurus. Code C143698.

A disorder characterized by obstruction of the nasal passage due to mucosal edema. 